\title{
The Critical Phenomena and Thermodynamics of the Reissner-Nordstrom-de Sitter Black Hole
}

\author{
Ren Zhao, ${ }^{1,2}$ Mengsen Ma, ${ }^{1,2}$ Huihua Zhao, ${ }^{1,2}$ and Lichun Zhang ${ }^{1,2}$ \\ ${ }^{1}$ Institute of Theoretical Physics, Shanxi Datong University, Datong 037009, China \\ ${ }^{2}$ Department of Physics, Shanxi Datong University, Datong 037009, China \\ Correspondence should be addressed to Ren Zhao; zhao2969@sina.com and Mengsen Ma; mengsenma@gmail.com
}

Received 25 October 2013; Revised 26 January 2014; Accepted 5 February 2014; Published 10 March 2014

Academic Editor: Deyou Chen

Copyright (C) 2014 Ren Zhao et al. This is an open access article distributed under the Creative Commons Attribution License, which permits unrestricted use, distribution, and reproduction in any medium, provided the original work is properly cited. The publication of this article was funded by SCOAP $^{3}$.

\begin{abstract}
It is wellknown that there are two horizons for the Reissner-Nordstrom-de Sitter spacetime, namely, the black hole horizon and the cosmological one. Both horizons can usually seem to be two independent thermodynamic systems; however, the thermodynamic quantities on both horizons satisfy the laws of black hole thermodynamics and are not independent. In this paper by considering the relations between the two horizons we give the effective thermodynamic quantities in Reissner-Nordstrom-de Sitter spacetime. The thermodynamic properties of these effective quantities are analyzed; moreover, the critical temperature, critical pressure, and critical volume are obtained. We also discussed the thermodynamic stability of Reissner-Nordstrom-de Sitter spacetime.
\end{abstract}

\section{Introduction}

Black hole physics, especially the black hole thermodynamics, refer directly to the theories of gravity, statistical physics, particle physics, field theory, and so forth. This makes the field concerned by many physicists [1-6]. Although the complete statistical description of black hole thermodynamics is still unclear, the research on the properties of black hole thermodynamics is prevalent, such as Hawking-Page phase transition [7], and critical phenomena. More interestingly, the research on the charged and nonrotating RN-AdS black hole shows that there exists a similar phase transition to the van der Waals-Maxwell vapor-liquid phase transition $[8,9]$.

Motivated by the AdS/CFT correspondence [10], where the transitions have been related with the holographic superconductivity $[11,12]$, the subject of the phase transitions of black holes in asymptotically anti-de Sitter (AdS) spacetime has received considerable attention [13-17]. The underlying microscopic statistical interaction of the black holes is also expected to be understood via the study of the gauge theory living on the boundary in the gauge/gravity duality.
Recently, by considering the cosmological constant correspond to pressure in general thermodynamic system, namely,

$$
P=-\frac{1}{8 \pi} \Lambda=\frac{3}{8 \pi} \frac{1}{l^{2}},
$$

the thermodynamic volumes in AdS and dS spacetime are obtained [18-24]. The studies on phase transition of black holes have aroused great interest [25-31]. Connecting the thermodynamic quantities of AdS black holes to $(P \sim V)$ in the ordinary thermodynamic system, the critical behaviors of black holes can be analyzed and the phase diagram like van der Waals vapor-liquid system can be obtained. This helps to further understand the black hole entropy, temperature, heat capacities, and so forth. It also has a very important significance in completing the geometric theory of black hole thermodynamics.

As is well known, there are black hole horizon and cosmological horizon in the appropriate range of parameters for de Sitter spacetime. Both horizons have thermal radiation, but with different temperatures. The thermodynamic quantities on both horizons satisfy the first law of thermodynamics, 
and the corresponding entropy fulfills the area formula [23, $32,33]$. In recent years, the research on the thermodynamic properties of de Sitter spacetime has drawn a lot of attention $[23,32-36]$. In the inflation epoch of early universe, the universe is a quasi-de Sitter spacetime. The cosmological constant introduced in de Sitter space may come from the vacuum energy, which is also a kind of energy. If the cosmological constant is the dark energy, the universe will evolve to a new de Sitter phase. To depict the whole history of evolution of the universe, we should have some knowledge on the classical and quantum properties of de Sitter space [23, 33, 37, 38].

Firstly, we expect the thermodynamic entropy to satisfy the Nernst theorem $[34,35,39]$. At present a satisfactory explanation to the problem in which the thermodynamic entropy of the horizon of the extreme de Sitter spacetime does not fulfill the Nernst theorem is still lacking. Secondly, when considering the correlation between the black hole horizon and the cosmological horizon whether the thermodynamic quantities in de Sitter spacetime still have the phase transition and critical behavior like in AdS black holes. Thus it is worthy of our deep investigation and reflection to establish a consistent thermodynamics in de Sitter spacetime.

Because the thermodynamic quantities on the black hole horizon and the cosmological one in de Sitter spacetime are the functions of mass $M$, electric charge $Q$, and cosmological constant $\Lambda$. The quantities are not independent of each other. Considering the relation between the thermodynamic quantities on the two horizons is very important for studying the thermodynamic properties of de Sitter spacetime. Based on the relation we give the effective temperature and pressure of Reissner-Nordstrom-de Sitter(R-NdS) spacetime and analyze the critical behavior of the equivalent thermodynamic quantities. It is shown that when considering the relation between the two horizons in $\mathrm{RN}$-dS spacetime there is the similar phase transition like the ones in van der Waals liquidgas system and charged AdS black holes.

The paper is arranged as follows. In Section 2 we introduce the Reissner-Nordstrom-de Sitter(R-NdS) spacetime and give the two horizons and corresponding thermodynamic quantities. In Section 3 by considering the relations between the two horizons we obtain the effective temperature and the equivalent pressure. In Section 4 the critical phenomena of effective thermodynamic quantities are discussed. Finally we discuss and summarize our results in Section 5 (we use the units $G_{n+1}=\hbar=k_{B}=c=1$ ).

\section{RN-dS Spacetime}

The line element of the R-N SdS black holes is given by [32]

$$
d s^{2}=-f(r) d t^{2}+f^{-1} d r^{2}+r^{2} d \Omega^{2},
$$

where

$$
f(r)=1-\frac{2 M}{r}+\frac{Q^{2}}{r^{2}}-\frac{\Lambda}{3} r^{2}
$$

The above geometry possesses three horizons: the black hole Cauchy horizon located at $r=r_{-}$, the black hole event horizon (BEH) located at $r=r_{+}$, and the cosmological event horizon (CEH) located at $r=r_{c}$, where $r_{c}>r_{+}>r_{-}$, the only real, positive zeroes of $f(r)=0$.

The equations $f\left(r_{+}\right)=0$ and $f\left(r_{c}\right)=0$ are rearranged to

$$
\begin{gathered}
Q^{2}=r_{+} r_{c}\left(1-\frac{r_{c}^{2}+r_{c} r_{+}+r_{+}^{2}}{3} \Lambda\right), \\
2 M=\left(r_{c}+r_{+}\right)\left(1-\frac{r_{c}^{2}+r_{+}^{2}}{3} \Lambda\right) .
\end{gathered}
$$

The surface gravity on the black hole horizon and the cosmological horizon is, respectively,

$$
\begin{aligned}
\kappa_{+} & =\left.\frac{1}{2} \frac{d f(r)}{d r}\right|_{r=r_{+}} \\
& =\frac{r_{+}^{2}-Q^{2}-r_{+}^{4} \Lambda}{2 r_{+}^{3}} \\
& =\frac{\left(r_{+}-r_{c}\right)}{2 r_{+}^{2}}\left(1-\frac{\left(r_{c}^{2}+2 r_{+} r_{c}+3 r_{+}^{2}\right)\left(r_{c} r_{+}-Q^{2}\right)}{r_{c} r_{+}\left(r_{c}^{2}+r_{+} r_{c}+r_{+}^{2}\right)}\right), \\
\kappa_{c} & =\left.\frac{1}{2} \frac{d f(r)}{d r}\right|_{r=r_{c}} \\
& =\frac{r_{c}^{2}-Q^{2}-r_{c}^{4} \Lambda}{2 r_{c}^{3}} \\
& =\frac{\left(r_{c}-r_{+}\right)}{2 r_{c}^{2}}\left(1-\frac{\left(r_{+}^{2}+2 r_{+} r_{c}+3 r_{c}^{2}\right)\left(r_{c} r_{+}-Q^{2}\right)}{r_{c} r_{+}\left(r_{c}^{2}+r_{+} r_{c}+r_{+}^{2}\right)}\right) .
\end{aligned}
$$

The thermodynamic quantities on the two horizons satisfy the first law of thermodynamics $[23,33,40,41]$ :

$$
\begin{gathered}
\delta M=\frac{\kappa_{+}}{2 \pi} \delta S_{+}+\Phi_{+} \delta Q+V_{+} \delta P, \\
\delta M=\frac{\kappa_{c}}{2 \pi} \delta S_{c}+\Phi_{c} \delta Q+V_{c} \delta P,
\end{gathered}
$$

where $S_{+}=\pi r_{+}^{2}, S_{c}=\pi r_{c}^{2}, \Phi_{+}=Q / r_{+}, \Phi_{c}=-\left(Q / r_{c}\right), V_{+}=$ $(4 \pi / 3) r_{+}^{3}, V_{c}=(4 \pi / 3) r_{c}^{3}, P=-(\Lambda / 8 \pi)$.

\section{Thermodynamic Quantity of RN-dS Spacetime}

In Section 2, we have obtained thermodynamic quantities without considering the relationship between the black hole horizon and the cosmological horizon. Because there are three variables $M, Q$, and $\Lambda$ in the spacetime, the thermodynamic quantities corresponding to the black hole horizon and the cosmological horizon are functional with respect to $M, Q$, and $\Lambda$. The thermodynamic quantities corresponding to the black hole horizon are related to the ones corresponding to the cosmological horizon. When the thermodynamic property of charged de Sitter spacetime is studied, we must consider the relationship with the two horizons. Recently, by studying Hawking radiation of de Sitter 
spacetime, $[42,43]$ obtained that the outgoing rate of the charged de Sitter spacetime which radiates particles with energy $\omega$ is

$$
\Gamma=e^{\Delta S_{+}+\Delta S_{c}},
$$

where $\Delta S_{+}$and $\Delta S_{c}$ are Bekenstein-Hawking entropy difference corresponding to the black hole horizon and the cosmological horizon after the charged de Sitter spacetime radiates particles with energy $\omega$. Therefore, the thermodynamic entropy of the charged de Sitter spacetime is the sum of the black hole horizon entropy and the cosmological horizon entropy:

$$
S=S_{+}+S_{c}
$$

Substituting (6) into (8), one can obtain

$$
\begin{aligned}
d S= & 2 \pi\left(\frac{1}{\kappa_{+}}+\frac{1}{\kappa_{c}}\right) d M \\
& -2 \pi\left(\frac{\varphi_{+}}{\kappa_{+}}+\frac{\varphi_{c}}{\kappa_{c}}\right) d Q+\frac{\pi}{3}\left(\frac{r_{+}^{3}}{\kappa_{+}}+\frac{r_{c}^{3}}{\kappa_{c}}\right) d \Lambda .
\end{aligned}
$$

For simplicity, we consider the case with constant. In this case the equation above turns into

$$
d S=2 \pi\left(\frac{1}{\kappa_{+}}+\frac{1}{\kappa_{c}}\right) d M+\frac{\pi}{3}\left(\frac{r_{+}^{3}}{\kappa_{+}}+\frac{r_{c}^{3}}{\kappa_{c}}\right) d \Lambda .
$$

From (4) and (5), we derive

$$
d r_{c}=\frac{d M}{r_{c} \kappa_{c}}+\frac{\left(r_{c}^{3} / 3\right)}{2 r_{c} \kappa_{c}} d \Lambda, \quad d r_{+}=\frac{d M}{r_{+} \kappa_{+}}+\frac{\left(r_{+}^{3} / 3\right)}{2 r_{+} \kappa_{+}} d \Lambda .
$$

Recently, the thermodynamic volume of RN-dS spacetime $[23,34]$ is given as

$$
V=\frac{4 \pi}{3}\left(r_{c}^{3}-r_{+}^{3}\right)
$$

Substituting (11) into (12), one gets

$$
d V=4 \pi\left(\frac{r_{c}}{\kappa_{c}}-\frac{r_{+}}{\kappa_{+}}\right) d M+\frac{2 \pi}{3}\left(\frac{r_{c}^{4}}{\kappa_{c}}-\frac{r_{+}^{4}}{\kappa_{+}}\right) d \Lambda .
$$

Substituting (13) into (10), one can derive the thermodynamic equation of thermodynamic quantities in de Sitter spacetime [34]:

$$
d M=T_{\mathrm{eff}} d S-P_{\mathrm{eff}} d V,
$$

where the effective temperature is

$$
\begin{aligned}
T_{\mathrm{eff}}= & \frac{\left(x^{4}+x^{3}-2 x^{2}+x+1\right)}{4 \pi r_{c} x(x+1)\left(x^{2}+x+1\right)} \\
& -\frac{Q^{2}}{4 \pi r_{c}^{3} x^{3}(x+1)\left(x^{2}+x+1\right)} \\
& \times\left(1+x+x^{2}-2 x^{3}+x^{4}+x^{5}+x^{6}\right) .
\end{aligned}
$$

The effective pressure is

$$
\begin{aligned}
P_{\mathrm{eff}}= & \frac{(1-x)\left(1+3 x+3 x^{2}+3 x^{3}+x^{4}\right)}{8 \pi r_{c}^{2} x(1+x)\left(1+x+x^{2}\right)^{2}} \\
& -\frac{Q^{2}(1-x)\left(1+2 x+3 x^{2}-3 x^{5}-2 x^{6}-x^{7}\right)}{8 \pi r_{c}^{4} x^{3}(1+x)\left(1-x^{3}\right)\left(1+x+x^{2}\right)},
\end{aligned}
$$

where $x:=r_{+} / r_{c}$ and $0<x<1$.

From (15) and (16), when $Q=0$, the effective temperature and pressure are both greater than zero. This fulfills the stable condition of thermodynamic equilibrium. If considering the black hole horizon and the cosmological one as independent of each other, because of the different radiant temperatures on the two horizons, the spacetime is instable.

Another problem of considering the black hole horizon and the cosmological one as independent of each other is that when the two horizons coincide, namely,

$$
r_{+/ c}^{2}=\frac{1 \pm \sqrt{1-4 Q^{2} \Lambda}}{2 \Lambda}=r_{0}^{2}
$$

from (4), the surface gravity $\kappa_{+/ c}=0$; thus the temperature on the black hole horizon and the temperature on the cosmological horizon are both zero. However, both horizons have nonzero area, which means that the entropy for the two horizons should not be zero. This conclusion is inconsistent with Nernst theorem. In the extreme case $r_{+/ c}^{2}=r_{0}^{2}$, the effective temperature from (15) is

$$
T_{\text {eff }}=\frac{1}{12 \pi r_{0}}\left(1-\frac{5 Q^{2}}{2 r_{0}^{2}}\right) .
$$

The effective pressure is

$$
P_{\text {eff }}=0 \text {. }
$$

In this case the volume-thermodynamic system becomes area-thermodynamic one. According to (19) the pressure of thermodynamic membrane is zero. However from (18), the temperature of thermodynamic membrane is nonzero. This can partly solve the problem that extreme de Sitter black holes do not satisfy the Nernst theorem, when $Q=0,(15)$ and (16) return to the known result [34].

\section{Critical Behaviour}

To compare with the van der Waals equation, we set $P_{\text {eff }} \rightarrow$ $P, v \rightarrow v$ and discuss the phase transition and the critical phenomena when $r_{c}$ is invariant. The van der Waals equation is

$$
\left(P+\frac{a}{v^{2}}\right)(v-\widetilde{b})=k T .
$$

Here, $v=V / N$ is the specific volume of the fluid, $P$ is its pressure, $T$ is its temperature, and $k$ is the Boltzmann constant.

Substituting (15) into (16), we obtain

$$
P_{\text {eff }}=T_{\text {eff }} \frac{B_{4}}{2 r_{c} B_{2}}+\frac{B_{2} B_{3}-B_{1} B_{4}}{8 \pi r_{c}^{2} x(1+x) B_{2}},
$$


where

$$
\begin{gathered}
B_{1}=\frac{1+x-2 x^{2}+x^{3}+x^{4}}{1+x+x^{2}} \\
B_{2}=\frac{1+x+x^{2}-2 x^{3}+x^{4}+x^{5}+x^{6}}{1+x+x^{2}} \\
B_{3}=\frac{1+2 x-2 x^{4}-x^{5}}{\left(1+x+x^{2}\right)^{2}} \\
B_{4}=\frac{1+2 x+3 x^{2}-3 x^{5}-2 x^{6}-x^{7}}{\left(1+x+x^{2}\right)^{2}} .
\end{gathered}
$$

According to (21), combing dimensional analysis [22] with (12), we conclude that we should identify the specific volume $v$ with

$$
v=r_{c}(1-x) .
$$

From the two equations

$$
\frac{\partial P_{\mathrm{eff}}}{\partial v}=0, \quad \frac{\partial^{2} P_{\mathrm{eff}}}{\partial v^{2}}=0,
$$

we first calculate the position of critical points. Then, one can derive

$$
\begin{gathered}
\left(\frac{\partial P_{\mathrm{eff}}}{\partial v}\right)_{T_{\mathrm{eff}}} \\
=-1 \times\left(8 \pi r c^{5}(1+x)\left(1+x+x^{2}\right)^{3}\right. \\
\left.\times\left(1+x+x^{2}-2 x^{3}+x^{4}+x^{5}+x^{6}\right)\right)^{-1} \\
\times\left[r c ^ { 2 } x ^ { 2 } \left(-6-21 x-38 x^{2}-45 x^{3}-30 x^{4}-10 x^{5}\right.\right. \\
\left.+6 x^{6}+7 x^{7}+4 x^{8}+x^{9}\right) \\
\times Q^{2} x\left(15+54 x+98 x^{2}+104 x^{3}+59 x^{4}+18 x^{5}\right. \\
\left.\left.-9 x^{6}-12 x^{7}-10 x^{8}-4 x^{9}-x^{10}\right)\right]=0 \\
\left(\frac{\partial^{2} P_{\mathrm{eff}}}{\partial v^{2}}\right)_{T_{\mathrm{eff}} \quad} \\
=1 \times\left(4 \pi r c^{6}(1+x)\left(1+x+x^{2}\right)^{4}\right. \\
\left.\times\left(1+x+x^{2}-2 x^{3}+x^{4}+x^{5}+x^{6}\right)^{2}\right)^{-1} \\
\times\left[\begin{array}{r}
-r c^{2} x\left(6+36 x+115 x^{2}+286 x^{3}+486 x^{4}\right. \\
+530 x^{5}+203 x^{6}-357 x^{7}-783 x^{8} \\
-854 x^{9}-666 x^{10}-375 x^{11}-127 x^{12} \\
\left.+3 x^{13}+32 x^{14}+18 x^{15}+6 x^{16}+x^{17}\right)
\end{array}\right.
\end{gathered}
$$

$$
\begin{aligned}
& +Q^{2}\left(30+165 x+456 x^{2}+837 x^{3}+1050 x^{4}\right. \\
& +903 x^{5}+255 x^{6}-720 x^{7}-1707 x^{8} \\
& +2113 x^{9}-1743 x^{10}-969 x^{11}-314 x^{12} \\
& +9 x^{13}+63 x^{14}+44 x^{15}+21 x^{16}+6 x^{17} \\
& \left.\left.+x^{18}\right)\right]=0 .
\end{aligned}
$$

When $r_{c}=1$, from (15), (16), and (25) one can obtain the position of critical point

$$
x^{c}=0.871992 .
$$

The critical electric charge, specific volume, temperature, and the critical pressure are, respectively,

$$
\begin{gathered}
Q^{c}=0.60395, \quad v^{c}=0.128008, \\
T^{c}=0.00436559, \quad p^{c}=0.0000145468 .
\end{gathered}
$$

In Figure 1 we give the figure of $P_{\text {eff }}$ with the change of $v$ at the constant effective temperature near the critical point.

To reflect the influence of $r_{c}$ on the spacetime, we set $r_{c}=$ 5 , from which we derived the position of the critical point

$$
x^{c}=0.871992 .
$$

The critical electric charge, specific volume, temperature, and the critical pressure are, respectively,

$$
\begin{array}{cl}
Q^{c}=3.01975, & v^{c}=0.640038, \\
T^{c}=0.000873119, & p^{c}=5.8187 \times 10^{-7} .
\end{array}
$$

The diagram of the effective $P_{\text {eff }}$ with the change of $v$ is depicted in Figure 2.

For the van der Waals fluid and the RN-AdS black hole, the relation $P_{c} v_{c} / T_{c}$ is a universal number and is independent of the charge Q. For the RN-dS black hole, according to the effective thermodynamic quantities, numerical calculation shows that $P_{c} v_{c} / T_{c}$ still has a $Q$-independent universal value. Certainly, the universal value is no more than $3 / 8$, but $\sim 0.0004265$

\section{Discussion and Conclusions}

From above we can find out that the value of $r_{c}$ does not influence the critical point $x$; namely, for the RN-dS spacetime, the position of critical point is irrelevant to the value of the cosmological horizon. This indicates that $x=$ $r_{c} / r_{+}$is fixed; however the critical effective temperature, critical volume, and critical pressure are dependent on the value of $r_{c}$. The critical effective temperature and pressure for the RN-dS system will decrease as the values of $r_{c}$ increase, while the critical electric charge and the critical volume will increase as the values of $r_{c}$ increase.

By Figures 1 and 2 , the $P_{\text {eff }}-v$ curve at constant temperature of $\mathrm{RN}$-dS spacetime is different from the ones of van der Waals equation and charged AdS black holes. 


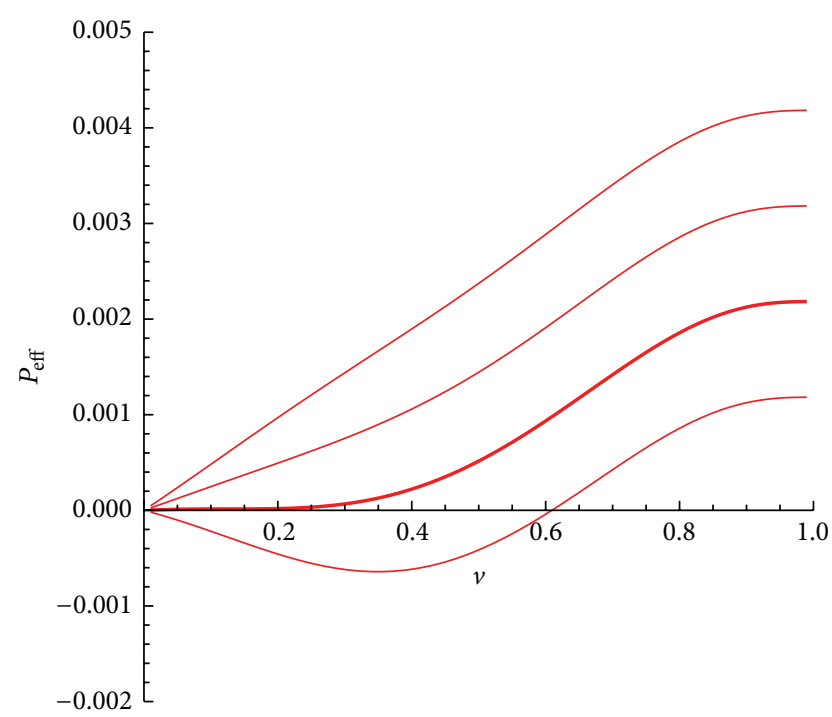

FIgURE 1: $P_{\text {eff }}-v$ diagram of RN-dS black holes. The temperature of isotherms decreases from top to bottom and corresponds to $T^{c}+$ $0.004, T^{c}+0.002, T^{c}, T^{\mathcal{C}}-0.002$.

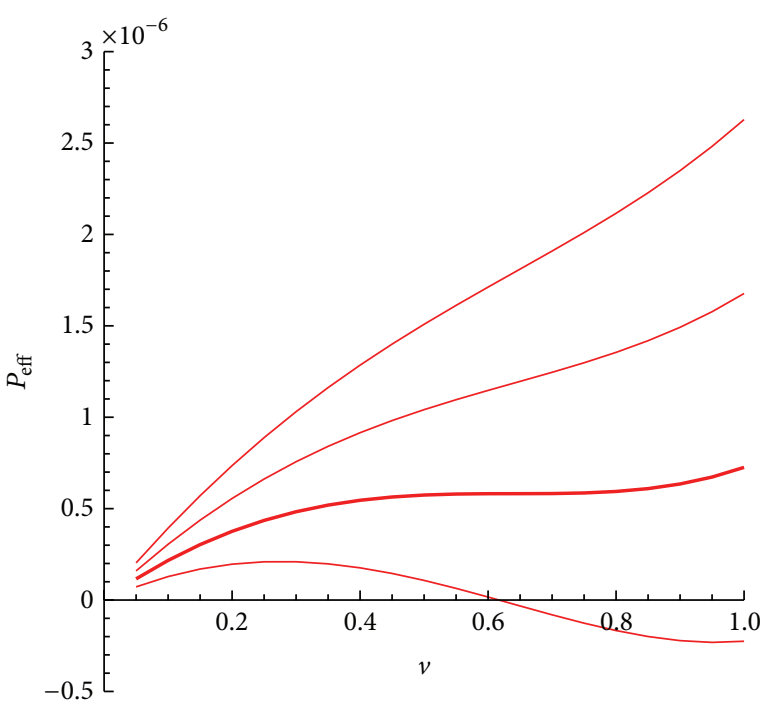

FIGURE 2: $P_{\text {eff }}-v$ diagram of RN-dS black holes. The temperature of isotherms decreases from top to bottom and corresponds to $T^{c}+$ $0.00004, T^{c}+0.00002, T^{c}, T^{c}-0.00002$.

(1) The first difference lies at the critical pressure $P_{\text {eff }}$ of RN-dS spacetime which increases as the volume increases at the constant temperature when $T_{\text {eff }}>T^{c}$, and the maximal value turns up at $x \rightarrow 0$, namely, de Sitter spacetime.

(2) The first difference is that the critical pressure $P_{\text {eff }}$ of $\mathrm{RN}$-dS spacetime may be negative at the constant temperature when $T_{\text {eff }}<T^{c}$, which means the system is not stable. From (15) and (16) we can obtain the parameters $x$ and $Q$ when the system is in the unstable state.
(3) On the $P_{\text {eff }}-v$ curves with $T_{\text {eff }}>T^{c}$ the system lies in a phase; on the $P_{\text {eff }}-v$ curves with $T_{\text {eff }}<T^{c}$ the same pressure will correspond to two different values of $x$, namely, two-phase coexistence region. Using the equal area criterion we can find out the proportion of the two phases for the RN-dS system.

From the above discussion, in RN-dS spacetime when considering the relation between the two horizons there is the similar phase transition like the one in van der Waals equation and charged AdS black holes. The reason is still unclear. This deserves further study. If the cosmological constant is just the dark energy, the universe will evolve into a new de Sitter phase. According to Figures 1 and 2 the RNdS system can evolve into de Sitter phase along isothermal curve. However, along the different isothermal curves, which processes will be the evolution of the universe to a new de Sitter phase needs further consideration according to the observations.

\section{Conflict of Interests}

The authors declare that there is no conflict of interests regarding the publication of this paper.

\section{Acknowledgments}

This work is supported by NSFC under Grant nos. 11175109 and 11075098 and by the Doctoral Sustentation Foundation of Shanxi Datong University (2011-B-03).

\section{References}

[1] J. D. Bekenstein, "Black holes and the second law," Lettere Al Nuovo Cimento, vol. 4, no. 15, pp. 737-740, 1972.

[2] J. D. Bekenstein, "Generalized second law of thermodynamics in black-hole physics," Physical Review D, vol. 9, no. 12, pp. 32923300, 1974.

[3] J. D. Bekenstein, "Extraction of energy and charge from a black hole," Physical Review D, vol. 7, no. 4, pp. 949-953, 1973.

[4] J. M. Bardeen, B. Carter, and S. W. Hawking, "The four laws of black hole mechanics," Communications in Mathematical Physics, vol. 31, pp. 161-170, 1973.

[5] S. W. Hawking, "Black hole explosions?" Nature, vol. 248, no. 5443, pp. 30-31, 1974.

[6] S. W. Hawking, "Particle creation by black holes," Communications in Mathematical Physics, vol. 43, no. 3, pp. 199-220, 1975.

[7] S. W. Hawking and D. N. Page, "Thermodynamics of black holes in anti-de Sitter space," Communications in Mathematical Physics, vol. 87, no. 4, pp. 577-588, 1983.

[8] A. Chamblin, R. Emparan, C. V. Johnson, and R. C. Myers, "Charged AdS black holes and catastrophic holography," Physical Review D, vol. 60, no. 6, Article ID 064018, 1999.

[9] A. Chamblin, R. Emparan, C. V. Johnson, and R. C. Myers, "Holography, thermodynamics, and fluctuations of charged AdS black holes," Physical Review D, vol. 60, no. 10, Article ID 104026, 1999.

[10] O. Aharony, S. S. Gubser, J. Maldacena, H. Ooguri, and Y. $\mathrm{Oz}$, "Large $N$ field theories, string theory and gravity," Physics Report, vol. 323, no. 3-4, pp. 183-386, 2000. 
[11] S. S. Gubser, "Breaking an Abelian gauge symmetry near a black hole horizon," Physical Review D, vol. 78, no. 6, Article ID 065034, 2008.

[12] S. A. Hartnoll, C. P. Herzog, and G. T. Horowitz, "Building an AdS/CFT superconductor," Physical Review Letters, vol. 101, Article ID 031601, 2008.

[13] A. Sahay, T. Sarkar, and G. Sengupta, "Thermodynamic geometry and phase transitions in Kerr-Newman-AdS black holes," Journal of High Energy Physics, vol. 2010, no. 4, article 118, 2010.

[14] A. Sahay, T. Sarkar, and G. Sengupta, "On the thermodynamic geometry and critical phenomena of AdS black holes," Journal of High Energy Physics, vol. 2010, no. 7, article 082, 2010.

[15] A. Sahay, T. Sarkar, and G. Sengupta, "On the phase structure and thermodynamic geometry of R-charged black holes," Journal of High Energy Physics, vol. 2010, no. 11, article 125, 2010.

[16] R. Banerjee, S. Ghosh, and D. Roychowdhury, "New type of phase transition in Reissner Nordström-AdS black hole and its thermodynamic geometry," Physics Letters B, vol. 696, no. 1-2, pp. 156-162, 2011.

[17] D. Kastor, S. Ray, and J. Traschen, "Enthalpy and the mechanics of AdS black holes," Classical and Quantum Gravity, vol. 26, no. 19, Article ID 195011, 2009.

[18] P. Brian Dolan, "The cosmological constant and the black hole equation of state," Classical and Quantum Gravity, vol. 28, Article ID 125020, 2011.

[19] S. Gunasekaran, D. Kubiznak, and R. B. Mann, "Extended phase space thermodynamics for charged and rotating black holes and Born-Infeld vacuum polarization," Journal of High Energy Physics, vol. 2012, article 110, 2012.

[20] B. P. Dolan, "Compressibility of rotating black holes," Physical Review D, vol. 84, Article ID 127503, 2011.

[21] M. Cvetic, G. W. Gibbons, D. Kubiznak, and C. N. Pope, "Black hole enthalpy and an entropy inequality for the thermodynamic volume," Physical Review D, vol. 84, Article ID 024037, 2011.

[22] D. Kubiznak and R. B. Mann, "P-V criticality of charged AdS black holes," Journal of High Energy Physics, vol. 1207, no. 33, 2012.

[23] B. P. Dolan, D. Kastor, D. Kubiznak, R. B. Mann, and J. Traschen, "Thermodynamic volumes and isoperimetric inequalities for de Sitter Black Holes," Physical Review D, vol. 87, Article ID 104017, 2013.

[24] B. P. Dolan, "Where is the PdV in the first law of black hole thermodynamics?" in Open Questions in Cosmology, J. Gonzalo Olmo, Ed., InTech, 2012, http://www.intechopen.com/ books/open-questions-in-cosmology/where-is-the-pdv-termin-the-first-law-of-black-hole-thermodynamics-.

[25] W. Shao-Wen and L. Yu-Xiao, "Critical phenomena and thermodynamic geometry of charged Gauss-Bonnet AdS black holes," Physical Review D, vol. 87, Article ID 044014, 2013.

[26] R.-G. Cai, L.-M. Cao, R.-Q. Yang et al., "PV criticality in the extended phase space of Gauss-Bonnet black holes in AdS space," Journal of High Energy Physics, vol. 2013, pp. 1-22, 2013.

[27] S. H. Hendi and M. H. Vahidinia, "Extended phase space thermodynamics and P-V criticality of black holes with nonlinear source," Physical Review D, vol. 88, Article ID 084045.

[28] A. Belhaj, M. Chabab, H. E. Moumni, and M. B. Sedra, "Critical behaviors of 3D black holes with a scalar hair," http://arxiv.org/abs/1306.2518.

[29] R. Zhao, H. H. Zhao, M. S. Ma, and L. C. Zhang, "On thermodynamics of charged and rotating asymptotically AdS black strings," The European Physical Journal C, vol. 73, no. 12, pp. 1-10, 2013.
[30] M. Bagher, J. Poshteh, B. Mirza, and Z. Sherkatghanad, "On the phase transition, critical behavior, and critical exponents of Myers-Perry black holes," Physical Review D, vol. 88, Article ID 024005, 2013.

[31] E. Spallucci and A. Smailagic, "Maxwell's equal area law for charged Anti-de Sitter black holes," Physics Letters B, vol. 723, no. 4-5, pp. 436-441, 2013.

[32] R.-G. Cai, "Cardy-Verlinde formula and thermodynamics of black holes in de Sitter spaces," Nuclear Physics B, vol. 628, no. 1-2, pp. 375-386, 2002.

[33] Y. Sekiwa, "Thermodynamics of de Sitter black holes: thermal cosmological constant," Physical Review D, vol. 73, no. 8, Article ID 084009, 2006.

[34] M. Urano and A. Tomimatsu, "Mechanical first law of black hole spacetimes with a cosmological constant and its application to the Schwarzschild-de Sitter spacetime," Classical and Quantum Gravity, vol. 26, no. 10, Article ID 105010, 2009.

[35] L. C. Zhang, H. F. Li, and R. Zhao, "Thermodynamics of the Reissner-Nordström-de Sitter black hole," Science China, vol. 54, no. 8, pp. 1384-1387, 2011.

[36] Y. S. Myung, "Thermodynamics of the Schwarzschild-de Sitter black hole: thermal stability of the Nariai black hole," Physical Review D, vol. 77, no. 10, Article ID 104007, 2008.

[37] R.-G. Cai, Physics, vol. 34, p. 555, 2005, (Chinese).

[38] S. Bhattacharya and A. Lahiri, "Mass function and particle creation in Schwarzschild-de Sitter spacetime," The European Physical Journal C, vol. 73, no. 12, pp. 1-10, 2013.

[39] R.-G. Cai, J.-Y. Ji, and K.-S. Soh, “Topological dilaton black holes," Classical and Quantum Gravity, vol. 15, no. 9, pp. 27832793, 1998.

[40] G. W. Gibbons, H. Lü, D. N. Page, and C. N. Pope, "Rotating black holes in higher dimensions with a cosmological constant," Physical Review Letters, vol. 93, no. 17, Article ID 171102, 2004.

[41] G. W. Gibbons, H. Lü, D. N. Page, and C. N. Pope, "The general Kerr-de Sitter metrics in all dimensions," Journal of Geometry and Physics, vol. 53, no. 1, pp. 49-73, 2005.

[42] R. Zhao, L.-C. Zhang, and H.-F. Li, "Hawking radiation of a Reissner-Nordström-de Sitter black hole," General Relativity and Gravitation, vol. 42, no. 4, pp. 975-983, 2010.

[43] Z. Ren, Z. Lichun, L. Huaifan, and W. Yueqin, "Hawking radiation of a high-dimensional rotating black hole," European Physical Journal C, vol. 65, no. 1, pp. 289-293, 2009. 

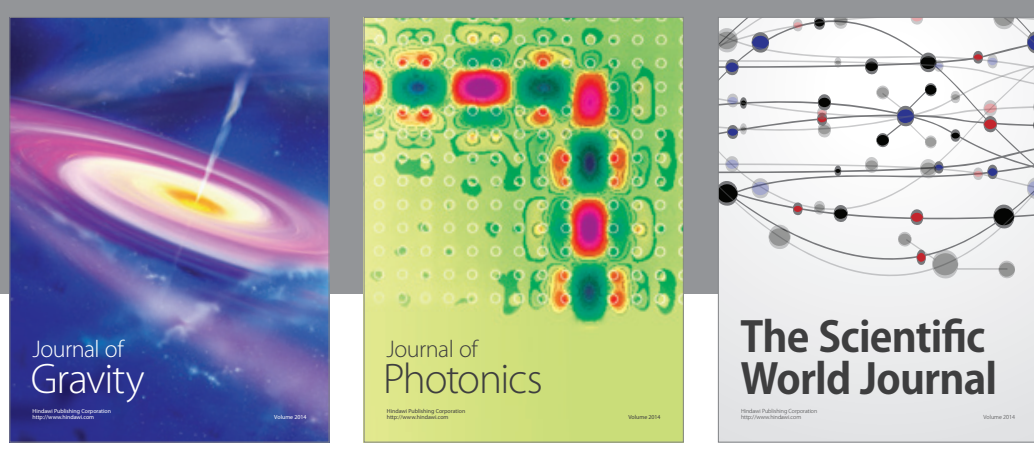

The Scientific World Journal
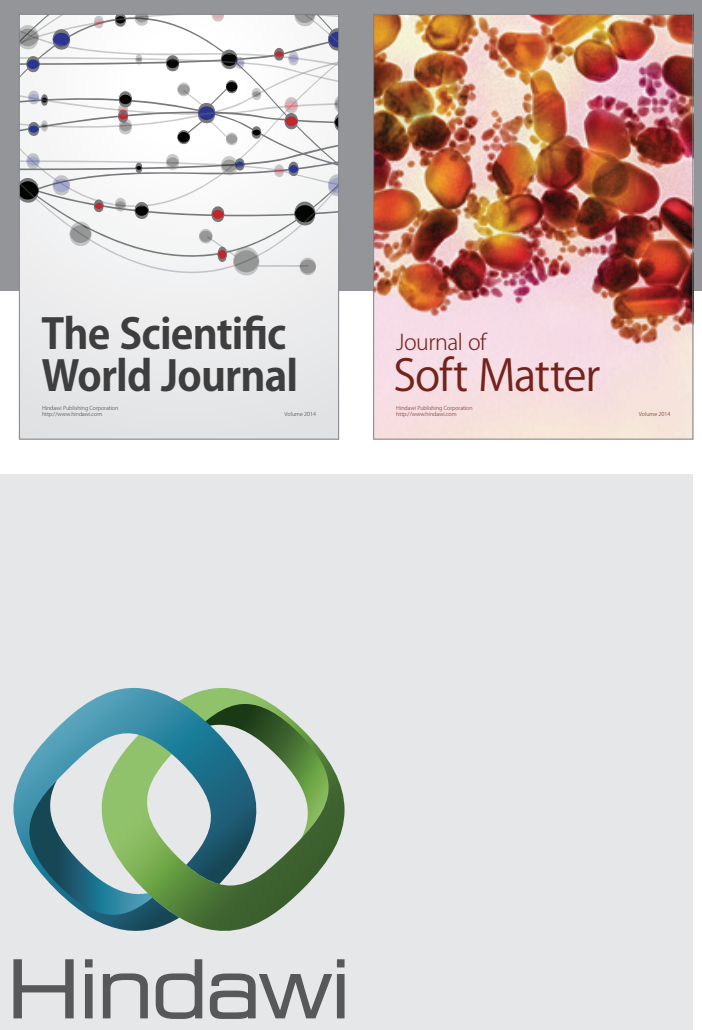

Submit your manuscripts at

http://www.hindawi.com

nternational Journal of

Statistical Mechanics
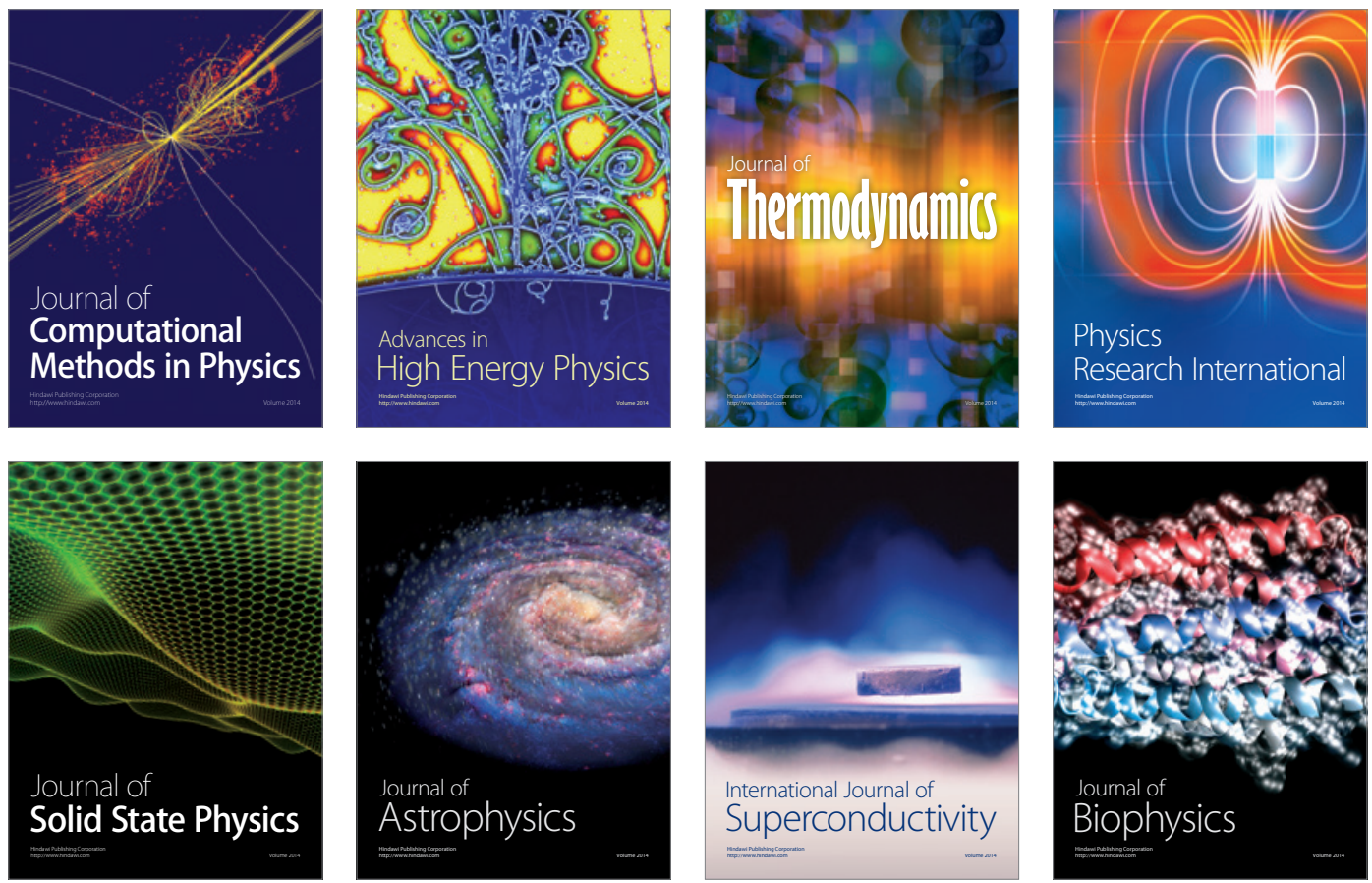
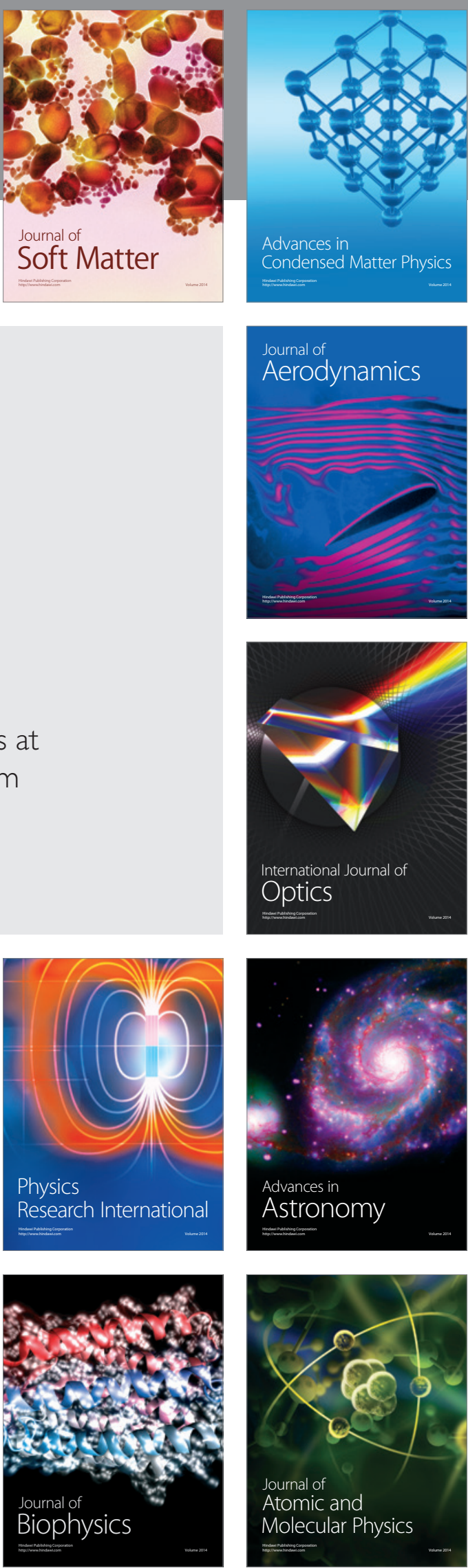